Conference Report

\title{
The Case for Nonlocal Modifications of Gravity
}

\author{
Richard P. Woodard
}

Department of Physics, University of Florida, Gainesville, FL 32611, USA; woodard@phys.ufl.edu

Received: 5 July 2018; Accepted: 6 August 2018; Published: 10 August 2018

\begin{abstract}
The huge amounts of undetected and exotic dark matter and dark energy needed to make general relativity work on large scales argue that we should investigate modifications of gravity. The only stable, metric-based and invariant alternative to general relativity is $f(R)$ models. These models can explain primordial inflation, but they cannot dispense with either dark matter or dark energy. I advocate nonlocal modifications of gravity, not as new fundamental theories but rather as the gravitational vacuum polarization engendered by infrared quanta produced during primordial inflation. I also discuss some of the many objections which have been raised to this idea.
\end{abstract}

Keywords: Nonlocal gravity; dark energy; dark matter; primordial inflation.

PACS: $04.50 . \mathrm{Kd} ; 95.35 .+\mathrm{d} ; 98.62 . \mathrm{g}$

\section{Introduction}

The case for nonlocal modifications of gravity is easy to make:

(1) The amount of "dark" stress-energy needed to make general relativity work strains credulity.

(2) The only metric-based, local, generally coordinate invariant and potentially stable alternative to general relativity is $f(R)$ models [1]. These can explain primordial inflation [2], but neither cosmic structures nor the current phase of cosmic acceleration.

(3) Although fundamental nonlocality seems problematic, nonlocal corrections to the effective field equations from loops of massless particles can give macroscopic effects, and those associated with the vast amount of inflationary particle production become nonperturbatively strong.

I discuss each of these points in Sections 2-4, respectively. Section 5 reviews four of the criticisms which have been raised to my work. My conclusions comprise Section 6.

\section{Shortcomings of Dark Matter and Energy}

Einstein's equation,

$$
G_{\mu v}=8 \pi G T_{\mu v},
$$

relates second and lower derivatives of the metric to the stress-energy tensor $T_{\mu \nu}$. No matter what metric $g_{\mu \nu}$ you want, there is a $T_{\mu \nu}$ which makes the equation true; general relativity is only tested when both sides are known. We know both sides of the equation for stars, but that ceases to be true on larger scales. Dark matter is needed to explain galaxies and galaxy clusters, while dark energy is invoked to explain the current phase of cosmic acceleration. Dark energy at a scale $10^{55}$ higher is the usual explanation for the early phase of accelerated expansion known as primordial inflation. In this section, I review why these explanations are problematic.

\subsection{The Willing Suspension of Disbelief}

Let me start with the sheer magnitude of exotic material which is required right now. We are told that only $4.6 \%$ of the current energy density of the universe can consist of anything we have ever 
detected in a laboratory. To explain cosmic structures, general relativity needs approximately $23 \%$ of the current energy density to consist of "dark matter" which has the equation of state of nonrelativistic matter but only interacts weakly. Explaining the current phase of cosmic acceleration requires that a whopping $72 \%$ of the current energy must consist of "dark energy" which has the equation of state of vacuum energy and interacts at most weakly.

None of this stuff has been seen, except gravitationally. The properties of dark energy do not require that it be visible in Earth-bound labs but dark matter should be, and it has not shown up. Two WIMP (Weakly Interacting Massive Partciles) searches of unprecedented sensitivity reported last year: neither the Xenon 1-ton experiment [3] nor PandaX-II [4] has detected anything. The continued failure to find dark matter has shaken the faith of even some passionate believers. To be sure, certain candidates such as Axions are still viable [5]. There is also very interesting recent work [6] on the old idea that dark matter might might not be exotic at all, but consists instead of normal matter which formed primordial black holes during inflation [7].

\subsection{Unexplained Regularities of Cosmic Structures}

For me, the real problem with dark matter is its failure to explain observed regularities in cosmic structures. These are well explained by Milgrom's MOdified Newtonian Dynamics (MOND) [8-10] which can be viewed as the static, weak field limit of a modified gravity theory [11,12]. Among the regularities it explains are [13]:

- The Baryonic Tully-Fisher Relation $v^{4}=a_{0} G M$ between the asymptotic rotational velocity $v$ and the baryonic mass $M$ of some structure, where $a_{0} \simeq 1.2 \times 10^{-10} \mathrm{~m} / \mathrm{s}^{2}$ [14];

- Milgrom's Law that dark matter always starts being necessary when the acceleration drops below $a_{0}[15] ;$

- $\quad$ Freeman's Law $G \Sigma<a_{0}$ for the surface density $\Sigma$ [16]; and

- Sancisi's Law that features in luminous matter follow features in rotation curves and vice versa [17].

Especially impressive is the recent work by McGaugh and collaborators on the universal relation which seems to exist between the observed radial acceleration and that predicted using only baryons [18,19]. This does not accord well with the idea that dark matter is five times more prevalent than baryonic matter. With general relativity plus dark matter one has to wonder, why is the baryonic matter tail wagging the dark matter dog?

The bottom line is that MOND provides too good a fit to evolved structures to be an accident. Either general relativity with dark matter approaches MOND as some kind of hitherto unrecognized attractor solution or else there is no dark matter and MOND represents the nonrelativistic, static limit of some modified gravity theory. Either possibility is fascinating, and I do not think anyone can honestly claim to know which is correct right now. Because I work in gravity, I have chosen to explore the second possibility.

\subsection{Fine Tuning Problems}

I think fine tuning is the worst problem for dark energy, and for primordial inflation. The usual explanation for both is general relativity with a minimally coupled scalar whose potential drives acceleration,

$$
\mathcal{L}=\frac{R \sqrt{-g}}{16 \pi G}-\frac{1}{2} \partial_{\mu} \varphi \partial_{\nu} \varphi g^{\mu \nu} \sqrt{-g}-V(\varphi) \sqrt{-g} .
$$

There is no question that this sort of model can support the required expansion histories because there is a closed form procedure for constructing the potential [1,20-24]. Suppose the desired geometry takes the form,

$$
d s^{2}=-d t^{2}+a^{2}(t) d \vec{x} \cdot d \vec{x} \quad \Longrightarrow \quad H(t) \equiv \frac{\dot{a}}{a}, \epsilon(t) \equiv-\frac{\dot{H}}{H^{2}}
$$


We assume the scalar depends only on time $\varphi=\varphi_{0}(t)$. The two nontrivial Einstein equations are,

$$
\begin{aligned}
3 H^{2} & =8 \pi G\left[\frac{1}{2} \dot{\varphi}_{0}^{2}+V\left(\varphi_{0}\right)\right], \\
-2 \dot{H}-3 H^{2} & =8 \pi G\left[\frac{1}{2} \dot{\varphi}_{0}^{2}-V\left(\varphi_{0}\right)\right] .
\end{aligned}
$$

Adding Equations (4) and (5) gives an equation we can solve for the scalar, up to an initial condition and a sign choice,

$$
-2 \dot{H}=8 \pi G \dot{\varphi}_{0}^{2} \Longrightarrow \varphi_{0}(t)=\varphi_{0}\left(t_{i}\right) \pm \int_{t_{i}}^{t} d s \sqrt{\frac{-2 \dot{H}(s)}{8 \pi G}} .
$$

Assuming $\dot{H}(t)$ is negative-definite, we can invert this (at least numerically) to determine the time as a function of the scalar $\tau(\varphi)$. Now subtract Equation (5) from Equation (4) and solve for the potential,

$$
V(\varphi)=\left.\frac{\dot{H}(t)+3 H^{2}(t)}{8 \pi G}\right|_{t=\tau(\varphi)}
$$

The construction of Equations (3)-(7) leaves no doubt that scalar potential models (Equation (2)) can support any expansion history with $\dot{H}(t)<0$, but we are left wondering, who ordered that? More quantitative questions abound:

- Why is $\varphi(t, \vec{x}) \sim \varphi_{0}(t)$ so spatially homogeneous?

- Why is $G^{2} V\left(\varphi_{0}\right) \sim 10^{-122}$ so small?

- Why is no fifth force observed?

For primordial inflation, the degree of fine tuning needed to get inflation to start, and the tendency to lose predictivity [25] has led to considerable angst within the community [26-28]. There is an additional problem associated with the need to couple the inflation $\varphi$ to ordinary matter to make reheating efficient. On de Sitter background $(\epsilon=0)$, the resulting cosmological Coleman-Weinberg potentials turn out to depend in a complicated way on the dimensionless ratio of $\varphi / H$. These potentials are not Planck-suppressed and they cannot be fine-tuned away because the factors of " $H$ " are not even local for a general metric [29]. This seems to have a disastrous effect on inflation [30].

I should mention that there are two reasonable alternatives to Equation (2) for primordial inflation which avoid some of the fine-tuning problems. One of these models employs the Higgs as the inflation, but with a huge conformal coupling [31]. The other is a modified gravity theory based on adding a large $R^{2}$ term to the Hilbert action [2].

\section{Options for Modifying Gravity}

Modified gravity theories can be classified based on the answers to three questions:

1. Is the gravitational force entirely carried by the metric or are other fields involved?

2. Is full general coordinate invariance preserved?

3. Are the field equations local or nonlocal?

In this section, I discuss metric-based modifications of gravity which preserve full general coordinate invariance. An important theorem restricts local, stable theories of this type to just $f(R)$ models [1]. I begin by explaining why $f(R)$ models cannot replace either dark energy or dark matter. I then review the problems associated with fundamental nonlocality.

\subsection{Problems with $f(R)$ Models}

I have already mentioned that an $f(R)$ model can give primordial inflation [2]. The same is not true for explaining the current phase of late time acceleration. The data tell us that the $\Lambda C D M$ 
expansion history seems to be correct [32,33]; however, the only stable $f(R)$ model which reproduces the $\Lambda$ CDM expansion history is $f(R)=R-2 \Lambda$ [34]. This means that any $f(R)$ model which replaces dark energy is bound to show discrepancies with the data at 0th order, without even worrying about perturbations.

To see the problem replacing dark matter, consider the physics of a static, spherically symmetric and nearly flat geometry,

$$
d s^{2}=-[1+b(r)] d t^{2}+[1+a(r)] d r^{2}+r^{2} d \Omega^{2} .
$$

Suppose $M(r)$ represents the mass enclosed within radius $r$. If this system is a low surface brightness galaxy within the MOND regime for all $r$, then the baryonic Tully-Fisher relation says the potential $b(r)$ must obey [35],

$$
v^{4}(r)=a_{0} G M(r)=\left[\frac{1}{2} r b^{\prime}(r)\right]^{2} .
$$

Because $M(r)$ is an integral over the mass density $\rho(r)$, we can recover what must be the weak field, static limit of the MOND equation for $b(r)$,

$$
\frac{\delta S}{\delta b(r)}=\frac{1}{32 \pi a_{0} G} \partial_{r}\left[r b^{\prime}(r)\right]^{2}-\frac{1}{2} r^{2} \rho(r)=0 .
$$

If we assume the $a$ equation is obeyed, the small $b$ expansion of the associated Lagrangian would be,

$$
\mathcal{L}=\frac{r^{2}}{16 \pi G}\left\{-\frac{b^{\prime 3}}{6 a_{0}}+O\left(b^{4}\right)\right\}-\frac{1}{2} r^{2} b \rho .
$$

Neither Equation (10) nor (11) can have come from any $f(R)$ model because the weak field expansion of the Ricci scalar is,

$$
R=-b^{\prime \prime}+\frac{2\left(a^{\prime}-b^{\prime}\right)}{r}+\frac{2 a}{r^{2}} .
$$

Note that the problem is fundamental, and has nothing to do with the weak field expansion. Ricci scalars have two derivatives, or factors of $1 / r$, whereas the desired field equation (10), has three derivatives.

\subsection{Problems with Fundamental Nonlocality}

I think it would be fair to say that Sir Isaac Newton disapproved of nonlocal equations of motion. He denounced it as so [36],

great an Absurdity that I believe no Man who has in philosophical Matters a competent Faculty of thinking can ever fall into it.

Now I know that some people at this conference, who do have a competent faculty of thinking, support fundamental nonlocality. Without engaging in Newton's vituperation, let me explain why I share the great man's doubts about the subject.

Ostrogradsky's theorem states that nondegenerate higher derivative models have extra degrees of freedom, essentially half of which carry negative kinetic energy [37]. When these sorts of theories have interactions among continuum fields, they develop a crazy time dependence in which the positive energy degrees of freedom become infinitely excited by infinitely exciting the negative energy ones. Some nonlocal theories can avoid this, but not the type favored by people here, which is based on entire functions of the derivative operator. An entire function is defined to converge to its Taylor series expansion, so we know one can view the theories of interest as the limits of sequences of ever-higher derivative models [38]. The theories in such a sequence become more unstable, not less, as the number of higher derivatives increases. In the fully nonlocal limit, one can specify the dynamical variable 
arbitrarily within any finite coordinate range, adjusting the variable outside this range to make the equation true. Assertions to the contrary are often based on working perturbatively in Euclidean momentum space. This amounts to assuming away the problem because the wild time dependence precludes the existence of temporal Fourier transforms in the first place.

People who claim to have solved the problem of extra, and unstable, initial value data sometimes ask me for an example of a system on which they might apply it. As it happens, I spent the better part of a year trying to come up with a good solution to the problem of using the scalar power spectrum of primordial inflation to reconstruct the first slow roll parameter $\epsilon$, regarded as a function of the number of e-foldings $n$, for the case where there are features. A simplified version of this problem takes the form of a linear integro-differential equation for $\ln [\epsilon(n)][39,40]$,

$$
\left[1+G(1) \partial_{n}\right] \ln [\epsilon(n)]-\int_{0}^{n} d m\left[\partial_{m}^{2}+3 \partial_{m}\right] \ln [\epsilon(m)] \times G\left(e^{m-n}\right)=f(n),
$$

where the function $G(x)$ is,

$$
G(x) \equiv \frac{1}{2}\left(x+x^{3}\right) \sin \left[\frac{2}{x}-2 \arctan \left(\frac{1}{x}\right)\right]
$$

We never did get a really satisfactory solution, precisely because of the Ostrogradskian instability. Those of you who think this is no issue, please solve my problem and then we can talk.

Before closing, I should mention the claims of another faction of those who believe in fundamental nonlocality, and also higher derivative theories. These people acknowledge the classical problem but assert that it can be evaded by clever alternate quantizations. The details do not matter much because all such claims suffer from the same problem of giving up the classical Correspondence Limit. Of course that must be the case because the classical theory has negative energy field configurations, whereas the spectrum of the alternate quantization does not. Physics is ultimately an experimental subject and if someone advanced this idea for anything other than gravity I would agree to let experiment decide the issue. However, the only low energy gravitational data we have, or ever will have, are from classical general relativity. It is a bad bargain to throw that away in the search for something you call "quantum gravity".

\section{Modified Gravity as Vacuum Polarization}

In criticizing fundamental nonlocality, it might be thought that I have undercut the case I wish to make. However, there is a completely acceptable type of nonlocality in the form of quantum corrections to the effective field equations. I first discuss how loops of massless particles can give macroscopic effects, even in flat space. Then, I discuss why primordial inflation might produce even stronger effects. This is followed by a review of corrections to electromagnetism and to gravitation which become nonperturbatively strong during a prolonged phase of primordial inflation. The section closes by reviewing the proposal that such effects might provide a model for primordial inflation.

\subsection{Macroscopic Nonlocality in Flat Space QED}

We all know how electron-positron loops cause electrodynamic forces to become stronger at short distances. Static corrections to the Coulomb potential $\Phi(r)$ of an electron are described by the nonlocal equation,

$$
-\nabla^{2}\left[\Phi(r)+\frac{1}{2 \pi^{2} r} \int_{0}^{\infty} d k k \sin (k r) \chi_{e}(k) \widetilde{\Phi}(k)\right]=-e \delta^{3}(\vec{r})
$$


Here, $\widetilde{\Phi}(k)$ is the spatial Fourier transform of $\Phi(r)$ and $\chi_{e}(k)$ is the one loop contribution to the electric susceptibility,

$$
\chi_{e}(k)=\delta \chi_{e}+\frac{4 \alpha}{\pi} \int_{0}^{1} d x x(1-x)\left\{\ln (2 \Lambda)-1-\frac{1}{2} \ln \left[m_{e}^{2}+x(1-x) k^{2}\right]\right\},
$$

where $\Lambda$ is the ultraviolet cutoff and $\delta \chi_{e}$ is the counterterm. As long as the electron mass $m_{e}$ is nonzero, we would choose $\delta \chi_{e}$ to make the susceptibility vanish at $k=0$,

$$
\chi_{e}(0)=0 \quad \Longrightarrow \quad \chi_{e}(k)=-\frac{2 \alpha}{\pi} \int_{0}^{1} d x x(1-x) \ln \left[1+x(1-x) \frac{k^{2}}{m_{e}^{2}}\right] .
$$

Of course, this means that there is no correction to the classical result for large $r$, however, the small $r$ potential experiences a logarithmic enhancement,

$$
r \lesssim \frac{1}{m_{e}} \quad \Longrightarrow \quad \Phi(r)=-\frac{e}{4 \pi r}\left[1+\frac{2 \alpha}{3 \pi} \ln \left(\frac{1}{m_{e} r}\right)+\ldots\right] .
$$

Now, suppose the electron mass vanished. In this case, we could no longer choose $\delta \chi_{e}$ to make the susceptibility vanish at $k=0$. We would instead choose some renormalization length scale $R$,

$$
m_{e}=0 \quad \Longrightarrow \quad \chi_{e}(k)=-\frac{2 \alpha}{\pi} \int_{0}^{1} d x x(1-x)\left[x(1-x) k^{2} R^{2}\right]
$$

In this case, the potential shows logarithmic corrections for all $r$,

$$
\forall r \Longrightarrow \Phi(r)=-\frac{e}{4 \pi r}\left[1+\frac{2 \alpha}{3 \pi} \ln \left(\frac{R}{r}\right)+\ldots\right] .
$$

At small $r$, Equation (20) exhibits the same enhancement as for a massive electron (Equation (18)). However, for large radius, the potential is weakened, and the effect becomes nonperturbatively strong for $r \gg R$. Because the effective coupling is weakened for large $r$, we can sum the sequence of leading logarithms to obtain a nonperturbative result for the large $r$ potential,

$$
\Phi(r) \longrightarrow-\frac{e}{4 \pi r} \times \frac{1}{\sqrt{1-\frac{4 \alpha}{3 \pi} \ln \left(\frac{R}{r}\right)}} .
$$

\subsection{Inflationary Particle Production}

Let us assume that inflation is driven by a minimally coupled scalar potential model (Equation (2)). The most transparent gauge is that of Salopek, Bond and Bardeen [41], in which the temporal condition sets $\varphi(t, \vec{x})=\varphi_{0}(t)$ and the spatial conditions are the transversality of the graviton. In this gauge, the metric components $g_{0 \mu}$ are constrained and the dynamical variables $\zeta(t, \vec{x})$ and $h_{i j}(t, \vec{x})$ appear in the spatial components,

$$
g_{i j}(t, \vec{x})=a^{2}(t) e^{2 \zeta(t, \vec{x})} \times\left[e^{h(t, \vec{x})}\right]_{i j} \quad, \quad h_{i i}(t, \vec{x})=0 .
$$

The quadratic part of the gauge fixed and constrained Lagrangian is,

$$
\mathcal{L}^{(2)}=\frac{\epsilon a^{3}}{8 \pi G}\left(\dot{\zeta}^{2}-\frac{1}{a^{2}} \vec{\nabla} \zeta \cdot \vec{\nabla} \zeta\right)+\frac{a^{3}}{64 \pi G}\left(\dot{h}_{i j} \dot{h}_{i j}-\frac{1}{a^{2}} h_{i j, k} h_{i j, k}\right) .
$$


Although the factors of $a(t)$ and $\epsilon(t)$ in Equation (23) break time translation invariance, spatial translation invariance is still present. This means that the scalar and the graviton fields can be decomposed into spatial plane waves. The equation of motion, Wronskian and asymptotic early time form for the scalar mode functions $v(t, k)$ are,

$$
\ddot{v}+\left(3 H+\frac{\dot{\epsilon}}{\epsilon}\right) \dot{v}+\frac{k^{2} v}{a^{2}}=0, v \dot{v}^{*}-\dot{v} v^{*}=\frac{i}{\epsilon a^{3}}, v \longrightarrow \frac{\exp \left[-i k \int_{t_{i}}^{t} \frac{d t^{\prime}}{a\left(t^{\prime}\right)}\right]}{\sqrt{2 k \epsilon(t) a^{2}(t)}} .
$$

The tensor mode functions $u(t, k)$ obey very similar relations,

$$
\ddot{u}+3 H \dot{u}+\frac{k^{2} u}{a^{2}}=0, u \dot{u}^{*}-\dot{u} u^{*}=\frac{i}{a^{3}}, u \longrightarrow \frac{\exp \left[-i k \int_{t_{i}}^{t} \frac{d t^{\prime}}{a\left(t^{\prime}\right)}\right]}{\sqrt{2 k a^{2}(t)}} .
$$

During inflation, $a(t)$ grows nearly exponentially, whereas $H(t)$ is almost constant. The result is that modes which are originally sub-horizon with $k>H(t) a(t)$ eventually experience first horizon crossing at $k=H\left(t_{k}\right) a\left(t_{k}\right)$. One can see from the mode equations (24) and (25) that the $k^{2} / a^{2}$ terms become irrelevant after horizon crossing, which causes $v(t, k)$ and $u(t, k)$ to approach constants with deviations falling off as $k^{2} / a^{2}$. These constants determine the scalar and tensor power spectra,

$$
\Delta_{\mathcal{R}}^{2}(k)=4 \pi G \times \frac{k^{3}}{2 \pi^{2}} \times|v(t, k)|_{t \gg t_{k}}^{2}, \Delta_{h}^{2}(k)=32 \pi G \times \frac{k^{3}}{2 \pi^{2}} \times 2 \times|u(t, k)|_{t \gg t_{k}}^{2} .
$$

The spatial translation invariance of Equation (23) means that spatially Fourier transformed fields with wave vector $\vec{k}$ behave as independent harmonic oscillators. However, the associated masses and frequencies are time dependent,

$$
\begin{aligned}
\zeta & \Longrightarrow m(t) \sim \epsilon(t) a^{3}(t), \quad \omega(t, k)=\frac{k}{a(t)}, \\
h_{i j} & \Longrightarrow m(t) \sim a^{3}(t) \quad, \quad \omega(t, k)=\frac{k}{a(t)} .
\end{aligned}
$$

The fact that spatial plane waves are independent harmonic oscillators means that the spectrum of energies at any instant of time is $\left(N+\frac{1}{2}\right) \hbar \omega(t, k)$. However, the time dependence of Equations (27) and (28) means that the energy eigenstates at one time do not remain eigenstates. The usual "vacuum" state is the one which was minimum energy $(N=0)$ in the distant past. The expectation value of the scalar and tensor energies at later times take the forms,

$$
\begin{aligned}
\left\langle\Omega\left|E_{\zeta}(t, k)\right| \Omega\right\rangle & =\frac{1}{2} \epsilon(t) a^{3}(t)\left[|\dot{v}(t, k)|^{2}+\frac{k^{2}}{a^{2}(t)}|v(t, k)|^{2}\right] \\
& \longrightarrow \frac{k}{a(t)} \times \frac{\pi \Delta_{\mathcal{R}}^{2}(k)}{4 G k^{2}} \times \epsilon(t) a^{2}(t) \\
\left\langle\Omega\left|E_{h}(t, k)\right| \Omega\right\rangle & =\frac{1}{2} a^{3}(t)\left[|\dot{u}(t, k)|^{2}+\frac{k^{2}}{a^{2}(t)}|u(t, k)|^{2}\right] \\
& \longrightarrow \frac{k}{a(t)} \times \frac{\pi \Delta_{h}^{2}(k)}{64 G k^{2}} \times a^{2}(t) .
\end{aligned}
$$

If we define occupation numbers based on these energies being $\left(\frac{1}{2}+N\right) \hbar \omega$, the numbers of inflationary scalars and gravitons with a single super-horizon wave vector $\vec{k}$ are,

$$
N_{\zeta}(t, k)=\frac{\pi \Delta_{\mathcal{R}}^{2}(k)}{4 G k^{2}} \times \epsilon(t) a^{2}(t) \quad, \quad N_{h}(t, k)=\frac{\pi \Delta_{h}^{2}(k)}{32 G k^{2}} \times a^{2}(t) .
$$


Of course, there are many wave vectors so the amount of inflationary particle production is truly staggering.

\subsection{Corrections to EM and GR During Inflation}

The best way of understanding quantum loop effects is through the action of classical physics on virtual particles. In view of the vast numbers (Equation (33)) of scalars and gravitons produced out of vacuum, it seems inevitable that quantum effects are strengthened during inflation. By solving the linearized effective field equations, we can see how inflationary scalars and gravitons modify particle kinematics and long range forces.

One studies how inflation affects electromagnetism by computing graviton and scalar contributions to the vacuum polarization $i\left[{ }^{\mu} \Pi^{\nu}\right]\left(x ; x^{\prime}\right)$ and then using this to quantum-correct Maxwell's equations,

$$
\partial_{v}\left[\sqrt{-g} g^{\nu \rho} g^{\mu \sigma} F_{\rho \sigma}(x)\right]+\int d^{4} x^{\prime}\left[{ }^{\mu} \Pi^{v}\right]\left(x ; x^{\prime}\right) A_{v}\left(x^{\prime}\right)=J^{\mu}(x) .
$$

The one loop graviton contribution to $i\left[{ }^{\mu} \Pi^{\nu}\right]\left(x ; x^{\prime}\right)$ was computed on de Sitter background [42] using the simplest gauge $[43,44]$. The result was employed to show that the electric fields of plane wave photons experience a secular enhancement [45],

$$
F_{0 i}^{11 \text { loop }}(t, k) \longrightarrow \frac{1}{\pi} G H^{2} \ln (a) \times F_{0 i}^{\text {tree }}(t, k) .
$$

Equation (34) also implies that the response to a point charge experiences a logarithmic running [46],

$$
\Phi(t, r)=\frac{Q}{4 \pi a r}\left\{1+\frac{G}{3 \pi a^{2} r^{2}}+\frac{1}{\pi} G H^{2} \ln (a H r)+O\left(G^{2}\right)\right\} .
$$

Note that both of these effects become nonperturbatively strong at late times and, in the case of Equation (36), at large $r$.

Quantum corrections to the linearized Einstein equation come from the graviton self-energy $-i\left[{ }^{\mu v} \Sigma^{\rho \sigma}\right]\left(x ; x^{\prime}\right)$,

$$
\sqrt{-g} \mathcal{L}^{\mu v \rho \sigma} h_{\rho \sigma}(x)-\int d^{4} x^{\prime}\left[{ }^{\mu v} \Sigma^{\rho \sigma}\right]\left(x ; x^{\prime}\right) h_{\rho \sigma}\left(x^{\prime}\right)=8 \pi G T^{\mu v}(x),
$$

where $\mathcal{L}^{\mu \nu \rho \sigma}$ is the Lichnerowicz operator in the appropriate background. The graviton self-energy was early computed in the simple gauge [47]. However, this result was not dimensionally regulated and fully renormalized, so it cannot be used in Equation (37). The equation was solved in the Hartree approximation to show that the electric curvature components of plane wave gravitons experience a secular de-enhancement [48],

$$
C_{0 i 0 j}^{1 \text { loop }}(t, k) \longrightarrow-\frac{8}{\pi} G H^{2} \ln (a) \times C_{0 i 0 j}^{\text {tree }}(t, k) .
$$

The contribution to $-i\left[{ }^{\mu v} \Sigma^{\rho \sigma}\right]\left(x ; x^{\prime}\right)$ from massless, minimally coupled scalars has been computed using dimensional regularization and fully renormalized [49]. This result shows no corrections to dynamical gravitons [50] but it does lead to a logarithmic decrease-in time and space-in the response to a point mass [51],

$$
\Psi(t, r)=-\frac{G M}{a r}\left\{1+\frac{G}{20 \pi a^{2} r^{2}}-\frac{G H^{2}}{10 \pi}\left[\frac{1}{3} \ln (a)+3 \ln (a H r)\right]+O\left(G^{2}\right)\right\} .
$$


Similar to their electromagnetic counterparts, perturbation theory breaks down for Equations (38) and (39).

\section{4. $\Lambda$-Driven Inflation}

We have just seen that quanta produced during inflation interact with external particles. It is obvious they must also interact with themselves. Because gravity is attractive, one might expect that they attract one another and that this mutual attraction should act as a sort of friction, slowing down the expansion rate. In line with the factors of $\ln (a)$ seen in Equations (35)-(39) one might expect that this friction grows with time as more and more of the newly produced quanta come into causal contact with one another. In addition, in line with Equations (35)-(39), we might expect that the secular slowing eventually becomes nonperturbatively strong.

No one knows what happens beyond perturbation theory but if we assume that secular slowing can eventually stop inflation, it becomes possible to imagine dispensing with the scalar altogether and driving inflation with a large, positive cosmological constant which is gradually screened by the build up of gravitational self-interaction between inflationary gravitons [52,53]. Such a model would solve many of the fine tuning problems associated with getting inflation to start, and to last long enough, and it would incidentally resolve the old problem of the cosmological constant $[54,55]$. It would also provide a unique model of inflation which made testable predictions-if only a way could be found to compute in the nonpertubative regime, as we did for massless QED in Equation (21).

\section{Answers to My Critics}

These effects in Equations (35)-(39) are astonishing, and the fact that they grow nonperturbatively strong is pregnant with possibilities for late time modifications of gravity. None of these results was easy to obtain. Some of the computations required the better part of a year's dedicated labor by me and/or collaborators. Thus, it is disheartening to watch our work facilely dismissed. For example, three of us attended the 12-week KITP program Quantum Gravity Foundations: UV to IR in 2015. Infrared quantum gravitational effects during primordial inflation is a subject on which we have a fair claim to being world experts-but the world is not interested. Shun-Pei Miao was allotted five minutes to summarize her year-long computation showing that inflationary gravitons excite fermions by an amount which eventually becomes nonperturbatively large [56,57]. Tomislav Prokopec was given ten minutes to review his one- and two-loop work on scalar quantum electrodynamics during inflation [58-60]. In this section, I discuss four of the reasons which my critics give for their sublime indifference.

\section{1. "Your Effects are Gauge Dependent"}

First, this objection does not apply to the screening of gravity (Equation (39)) caused by loops of massless, minimally coupled scalars [49,51]. Gauge dependence requires that a graviton propagator enter the loop, and none does in that case. However, the effect on the Newtonian potential (Equation (39)) shows the same fractional correction of $G H^{2} \ln (a H r)$ that gravitons make to the Coulomb potential in Equations (36). I have already invoked Coleridge's famous comment on the willing suspension of disbelief, which surely applies to dismissing Equation (36) as a gauge artifact while (grudgingly) admitting the reality of Equation (39). A second, but closely related point concerns the fractional corrections of $G / a^{2} r^{2}$ which are visible in both Equations (36) and (39). These are nothing but the de Sitter descendants, with $r \rightarrow a(t) r$, of flat space corrections which were computed long ago [61].

My third comment concerns the physics of kinematical changes in photons (Equation (35)) and gravitons (Equation (38)). Without regard to the details of the computations, the vast numbers of particles produced during inflation must scatter external photons and gravitons to some extent. This is not some sort of gauge chimera; in flat space background, it is the basis of the proposal to use pulsar arrival times to detect gravitational radiation [62]. The eventual breakdown of perturbation theory 
evident in Equations (35) and (38) has a very simple origin: the longer an external photon or graviton propagates the more it should be affected. The numerical coefficients might be suspect, but the general trend must occur.

Of course computational details do matter because we want gauge independent results for the numerical coefficients. My fourth comment is that years of study have paid off in providing both a physical explanation for why the effective field equations are gauge dependent and a procedure for eliminating this gauge dependence [63]. Gauge dependence arises because some physical source disturbs the effective field, and some physical observer measures the disturbance. The source and observer interact with quantum gravity, as does everything, and we have not described a physical process unless we include this interaction. As might be expected, few of the source and observer details matter much. For example, it does not matter that the observer had blue eyes or brown.

Miao, Prokopec (who were granted $15 \mathrm{~min}$ at a 12-week program!) and I worked out an explicit example in flat space background concerning the one graviton loop correction to the exchange potential of a massless, minimally coupled scalar in the two-parameter family of covariant gauge fixing functions [63],

$$
\mathcal{L}_{G F}=-\frac{1}{2 a} \eta^{\mu v} F_{\mu} F_{v} \quad, \quad F_{\mu}=\eta^{\rho \sigma}\left(h_{\mu \rho, \sigma}-\frac{b}{2} h_{\rho \sigma, \mu}\right) .
$$

The linearized effective field equation can be expressed in terms of a self-mass-squared $M^{2}\left(x ; x^{\prime}\right)$,

$$
\partial^{2} \varphi(x)-\int d^{4} x^{\prime} M^{2}\left(x ; x^{\prime}\right) \varphi\left(x^{\prime}\right)=J(x) \text {. }
$$

Ignoring the source and observer, our result for $M^{2}\left(x ; x^{\prime}\right)$ in the gauge of Equation (40) takes the form of a gauge dependent constant times a function of spacetime whose form is fixed by Poincaré invariance and dimensionality,

$$
\begin{aligned}
-i M^{2}\left(x ; x^{\prime}\right) & =\mathcal{C}_{0}(a, b) \times \frac{G \partial^{6}}{4 \pi^{3}}\left[\frac{\ln \left(\mu^{2} \Delta x^{2}\right)}{\Delta x^{2}}\right], \Delta x^{2} \equiv\left(x-x^{\prime}\right)^{2} \\
\mathcal{C}_{0}(a, b) & =+\frac{3}{4}-\frac{3}{4} \times a-\frac{3}{2} \times \frac{1}{b-2}+\frac{3}{4} \times \frac{(a-3)}{(b-2)^{2}} .
\end{aligned}
$$

The fact that $\mathcal{C}_{0}(a, b)$ can be made to vary from $-\infty$ to $+\infty$ would provide my critics justification to condemn the whole exercise as nonsense. However, they would be wrong! By including quantum gravitational correlations from the source and observer one gets additional contributions having the same spacetime dependence as Equation (42) but with different gauge dependent coefficients. Table 1 summarizes the results and demonstrates the satisfying cancellation of all dependence on the parameters $a$ and $b$ [63]. Steven Weinberg relates how post-renormalization physicists used to quip, “just because something diverges doesn't mean it's zero". In the same vein, I hope people will now admit, "just because something depends on the gauge doesn't mean it's zero!".

Table 1. The gauge dependent factors $C_{i}(a, b)$ for each contribution to the self-mass-squared.

\begin{tabular}{cccccc}
\hline $\boldsymbol{i}$ & $\mathbf{1}$ & $\boldsymbol{a}$ & $\frac{\mathbf{1}}{\boldsymbol{b - 2}}$ & $\frac{(\boldsymbol{a}-3)}{(\boldsymbol{b}-2)^{2}}$ & Description \\
\hline 0 & $+\frac{3}{4}$ & $-\frac{3}{4}$ & $-\frac{3}{2}$ & $+\frac{3}{4}$ & scalar exchange \\
1 & 0 & 0 & 0 & +1 & vertex-vertex \\
2 & 0 & 0 & 0 & 0 & vertex-source,observer \\
3 & 0 & 0 & +3 & -2 & vertex-scalar \\
4 & $+\frac{17}{4}$ & $-\frac{3}{4}$ & 0 & $-\frac{1}{4}$ & source-observer \\
5 & -2 & $+\frac{3}{2}$ & $-\frac{3}{2}$ & $+\frac{1}{2}$ & scalar-source,observer \\
\hline Total & +3 & 0 & 0 & 0 & \\
\hline
\end{tabular}


Resolving the gauge problem for effective field equations is not enough. The correct generalization of the power spectrum is still unclear [64] but the answer may be gauge invariant correlators. There is interesting recent work on these by Markus Fröb and collaborators [65-67]. An invariant measure of the local expansion rate has also been proposed [68], and its one-loop renormalization on de Sitter background has been accomplished [69].

\section{2. "IR Gravitons Have Small Curvature"}

Each graviton mode has a constant wave vector $\vec{k}$, whose physical wave number $k / a(t)$ redshifts as the universe expands. During a sufficiently long epoch of inflation, the physical wave number eventually falls below the almost constant Hubble parameter $H(t)$, an event which is known as first horizon crossing. After inflation, the product $a(t) H(t)$ falls off, so modes can experience a second horizon crossing. Some of my critics appear to believe that only sub-horizon gravitons are physical, so that inflationary gravitons literally pass out of existence between first and second crossings. This is nonsense. The curvatures of super-horizon gravitons become small exponentially fast, but they never touch zero, and the exponentially growing numbers of super-horizon gravitons (Equation (33)) can compensate small individual curvatures.

Another point of great relevance is that the curvatures of inflationary gravitons are not always small; they start out large and then redshift. General relativity on asymptotically flat space has a well-known nonlinear memory effect [70] in which the passage of a gravitational wave leaves a permanent displacement in test observers. After the wave has passed, the observers' curvature is zero, yet the passage of the wave had an effect. How can it be argued that the continual redshift of gravitons from the ultraviolet to the infrared during inflation can have no effect?

I seem to be re-contesting the same battles which were fought last century over the fact that charged quantum particles couple to the undifferentiated vector potential, not the field strength. There never was any doubt about this, but stubborn physicists for years resisted the obvious conclusion that constant vector potentials could, under certain circumstances, engender physical effects. However, nature pays no attention to human prejudices and an experiment was eventually proposed [71,72] whose result [73] is taught in undergraduate quantum mechanics. In the same sense, matter, and gravity itself, couple to the undifferentiated metric, not to the curvature. Hence, there must be cases in which physical effects can occur even for zero curvature.

Finally, one must distinguish between infrared divergences and infrared effects such as secular growth (Equation (35)) and logarithmic running (Equation (36)). Infrared divergences derive from infinite numbers of gravitons being super-horizon at the start of inflation. A compelling case has been made that these would be subsumed into the background of any local observer and have no effect [74]. On the other hand, infrared effects are caused by gravitons which were initially sub-horizon and experienced first horizon crossing during inflation. It is not legitimate to regard these are having always been part of the background. Of course, it might still have been correct, as my critics insisted. I am a big believer in checking things when I can figure out how to do it. When I finally figured out how to check this belief, the result is that subsuming infrared gravitons into the background can neither eliminate secular growth [75] nor changes in the local expansion rate [76].

\section{3. "Your Effects Are Not Observable"}

Some claim that the cosmological horizon precludes local observers from perceiving effects such as Equations (35)-(39) during inflation. To see how a local observer could perceive the secular enhancement of photons (Equation (35)) consider setting off a flash between reflecting mirrors at fixed, sub-horizon physical distances from one another, and monitoring the field strength as the signal reflects back and forth. The logarithmic running of Equation (36) could be observed using one neutral and two charged particles. Release the two charges at rest from one another, with the neutral particle also released from rest, next to one of the charges. Then, even after the two charges are no longer in causal contact, the effect of their mutual attraction could be followed by measuring the separation 
of the nearby charge from the neutral particle. These sorts of experiments could at least be done for a while.

Other critics claim that Equations (35)-(39) can have no effect now. These people leave me wondering what part of the phrase "nonperturbatively strong" they do not understand. The burden of my message is that modifications to gravity now might derive from interactions between the vast numbers (Equation (33)) of scalars and gravitons produced during inflation. The effects of Equations (35)-(39) all grow to eventually become nonperturbatively strong during a prolonged period of inflation. The fact that I cannot yet sum up the series of large logarithms to exhibit their late time limits is no reason for claiming that those limits are unobservable.

\section{4. "Your Calculations Are Difficult"}

No one enjoys being attacked, but I have tried very hard to understand my critics and to honestly address their concerns. In a well-known case, I publicly renounced a previous opinion [77] based on this sort of engagement [78]. However, whining about the complexity of perturbative quantum gravity on de Sitter background is not a serious objection, although it is sadly frequent, and I can only urge this class of critics to grow up!

\section{Conclusions}

This article has been devoted to making the case for nonlocal modifications of gravity. In these concluding remarks, I briefly review some of the models I have explored. I apologize to the many others whose work on such nonlocal models I do not discuss [79-117].

As I have explained, nonlocal modifications of gravity can come from quantum loop effects that grew nonperturbatively strong during primordial inflation. Although one can follow some of these effects while they are still small, there is not yet any way to compute past the breakdown of perturbation theory, as we were able to do for the Coulomb potential in Equation (21) of massless QED in flat space. However, the putative inflationary origin does explain two things that would otherwise seem unnatural:

- There is an initial value surface upon which the initial conditions of inverse differential operators can be defined.

- Modifications of gravity are expected on large distances, not small ones.

We seek to guess the most cosmologically significant part of the gravitational effective action. What happens perturbatively should serve as a guide. One can see from Equations (35)-(39) that secular growth on de Sitter background resides in factors of $\ln [a(t)]$. A simple nonlocal scalar which reproduces this is [118],

$$
\left.\frac{1}{\square} R\right|_{\mathrm{dS}} \longrightarrow-4 \ln [a(t)],
$$

where $\square \equiv \frac{1}{\sqrt{-g}} \partial_{\mu}\left(\sqrt{-g} g^{\mu v} \partial_{v}\right)$ is the scalar d'Alembertian. Thus, it might be reasonable to expect that modifications involve an algebraic function $f\left(\frac{1}{\square} R\right)$. Models of this type have been proposed to study $\Lambda$-driven inflation [20,119], metric realizations of MOND [120], and late time acceleration [121-123]. Although this simple ansatz is not satisfactory for MOND [124], it does describe an interesting model for ending primordial inflation, generating density perturbations and then reheating to go quiescent into the epoch of radiation domination [125]. With some elaborations, it might even describe late time acceleration [126-128]. The simple ansatz also offers many advantages in describing late time acceleration because:

- $\quad$ Unlike $R \longrightarrow f(R)$ models, theories involving $R \longrightarrow R f\left(\frac{1}{\square} R\right)$ can be chosen to exactly reproduce the $\Lambda \mathrm{CDM}$ expansion history [129].

- Because $\frac{1}{\square} R$ is negative for cosmology and positive for gravitationally bound systems, it is trivial to choose the function $f\left(\frac{1}{\square} R\right)$ to avoid solar system constraints.

- The scalar $\frac{1}{\square} R$ is dimensionless so it requires no small mass. 
- During radiation domination $R=0$, so the onset of modifications is postponed until late in cosmological history.

- Even after matter domination, the scalar $\frac{1}{\square} R$ only grows logarithmically with time, postponing the onset to even later times.

- Perturbing the model to study structure formation [130-132] actually agrees better with the data than general relativity $[133,134]$.

A more elaborate nonlocal model involving an algebraic function of a different scalar has been devised to reproduce MOND pheneomenology for gravitationally bound systems [35]. Because this is a complete, metric theory of gravity, it can be applied to cosmology similar to general relativity $[135,136]$. The algebraic function can be chosen to reproduce most of the $\Lambda$ CDM expansion history, and even offers a serendipitous explanation for the tension between low redshift and high redshift determinations of the Hubble constant [137]. However, perturbations about the cosmological background do not correctly describe structure formation [138]. I suspect that the problem can be resolved by making the numerical coincidence $c H_{0} \simeq 2 \pi a_{0}$ dynamical.

Funding: This work was partially supported by NSF grants PHY-1506513 and PHY-1806218, and by the Institute for Fundamental Theory at the University of Florida.

Acknowledgments: The author is grateful for conversations and correspondence with S. Deser, E. Kiritsis and N. C. Tsamis.

Conflicts of Interest: The authors declare no conflict of interest.

\section{References}

1. Woodard, R.P. Avoiding dark energy with $1 / \mathrm{r}$ modifications of gravity. Lect. Notes Phys. 2007, 720, 403-433. [CrossRef]

2. Starobinsky, A.A. A New Type of Isotropic Cosmological Models Without Singularity. Phys. Lett. B 1980, 91, 99-102. [CrossRef]

3. Aprile, E.; Aalbers, J.; Agostini, F.; Alfonsi, M.; Amaro, F.; Anthony, M.; Arneodo, F.; Barrow, P.; Baudis, L.; Bauermeister, B.; et al. First Dark Matter Search Results from the XENON1T Experiment. Phys. Rev. Lett. 2017, 119, 181301. [CrossRef] [PubMed]

4. Cui, X.; Abdukerim, A.; Chen, W.; Chen, X.; Chen, Y.; Dong, B.; Fang, D.; Fu, C.; Giboni, K.; Giuliani, F.; et al. Dark Matter Results From 54-Ton-Day Exposure of PandaX-II Experiment. Phys. Rev. Lett. 2017, 119, 181302. [CrossRef] [PubMed]

5. Du, N.; Force, N.; Khatiwada, R.; Lentz, E.; Ottens, R.; Rosenberg, L.; Rybka, G.; Carosi, G.; Woollett, N.; Bowring, D.; et al. A Search for Invisible Axion Dark Matter with the Axion Dark Matter Experiment. Phys. Rev. Lett. 2018, 120, 151301. [CrossRef] [PubMed]

6. García-Bellido, J.; Clesse, S.; Fleury, P. Primordial black holes survive SN lensing constraints. Phys. Dark Univ. 2018, 20, 95-100. [CrossRef]

7. Dolgov, A.; Silk, J. Baryon isocurvature fluctuations at small scales and baryonic dark matter. Phys. Rev. D 1993, 47, 4244-4255. [CrossRef]

8. Milgrom, M. A Modification of the Newtonian dynamics as a possible alternative to the hidden mass hypothesis. Astrophys. J. 1983, 270, 365-370. [CrossRef]

9. Milgrom, M. A Modification of the Newtonian dynamics: Implications for galaxies. Astrophys. J. 1983, 270, 371-389. [CrossRef]

10. Milgrom, M. A modification of the Newtonian dynamics: Implications for galaxy systems. Astrophys. J. 1983, 270, 384. [CrossRef]

11. Sanders, R.H.; McGaugh, S.S. Modified Newtonian dynamics as an alternative to dark matter. Ann. Rev. Astron. Astrophys. 2002, 40, 263-317. [CrossRef]

12. Famaey, B.; McGaugh, S. Modified Newtonian Dynamics (MOND): Observational Phenomenology and Relativistic Extensions. Living Rev. Rel. 2012, 15, 10. [CrossRef] [PubMed]

13. Sanders, R.H. From dark matter to MOND. arXiv 2008, arXiv:0806.2585.

14. McGaugh, S.S. The Baryonic Tully-Fisher relation of galaxies with extended rotation curves and the stellar mass of rotating galaxies. Astrophys. J. 2005, 632, 859-871. [CrossRef] 
15. Kaplinghat, M.; Turner, M.S. How cold dark matter theory explains Milgrom's law. Astrophys. J. 2002, 569, L19-L22. [CrossRef]

16. Freeman, K.C. On the disks of spiral and SO Galaxies. Astrophys. J. 1970, 160, 811. [CrossRef]

17. Sancisi, R. The Visible matter-Dark matter coupling. IAU Symp. 2004, 220, 233-240. [CrossRef]

18. McGaugh, S.; Lelli, F.; Schombert, J. Radial Acceleration Relation in Rotationally Supported Galaxies. Phys. Rev. Lett. 2016, 117, 201101. [CrossRef] [PubMed]

19. Lelli, F.; McGaugh, S.S.; Schombert, J.M.; Pawlowski, M.S. One Law to Rule Them All: The Radial Acceleration Relation of Galaxies. Astrophys. J. 2017, 836, 152. [CrossRef]

20. Tsamis, N.C.; Woodard, R.P. Nonperturbative models for the quantum gravitational back reaction on inflation. Ann. Phys. 1998, 267, 145-192. [CrossRef]

21. Saini, T.D.; Raychaudhury, S.; Sahni, V.; Starobinsky, A.A. Reconstructing the cosmic equation of state from supernova distances. Phys. Rev. Lett. 2000, 85, 1162-1165. [CrossRef] [PubMed]

22. Padmanabhan, T. Accelerated expansion of the universe driven by tachyonic matter. Phys. Rev. D 2002, 66, 021301. [CrossRef]

23. Nojiri, S.; Odintsov, S.D. Unifying phantom inflation with late-time acceleration: Scalar phantom-non-phantom transition model and generalized holographic dark energy. Gen. Rel. Grav. 2006, 38, 1285-1304. [CrossRef]

24. Guo, Z.K.; Ohta, N.; Zhang, Y.Z. Parametrizations of the dark energy density and scalar potentials. Mod. Phys. Lett. A 2007, 22, 883-890. [CrossRef]

25. Ijjas, A.; Steinhardt, P.J.; Loeb, A. Inflationary paradigm in trouble after Planck2013. Phys. Lett. B 2013, 723, 261-266. [CrossRef]

26. Guth, A.H.; Kaiser, D.I.; Nomura, Y. Inflationary paradigm after Planck 2013. Phys. Lett. B 2014, 733, $112-119$. [CrossRef]

27. Linde, A. Inflationary Cosmology after Planck. In Post-Planck Cosmology; Deffayet, C., Peter, P., Wandelt, B., Zaldarriaga, M., Cugliandolo, L.F., Eds.; Oxford University Press: Oxford, UK, 2015.

28. Ijjas, A.; Steinhardt, P.J.; Loeb, A. Inflationary schism. Phys. Lett. B 2014, 736, 142-146. [CrossRef]

29. Miao, S.P.; Woodard, R.P. Fine Tuning May Not Be Enough. J. Cosmol. Astropart. Phys. 2015, $1509,022$. [CrossRef]

30. Liao, Z.H.; Miao, S.P.; Woodard, R.P. Cosmological Coleman-Weinberg Potentials and Inflation. arXiv 2018, arXiv:1806.02533.

31. Bezrukov, F.L.; Shaposhnikov, M. The Standard Model Higgs boson as the inflaton. Phys. Lett. B 2008, 659, 703-706. [CrossRef]

32. Abbott, T.M.C.; Abdalla, F.B.; Alarcon, A.; Aleksić, J.; Allam, S.; Allen, S.; Amara, A.; Annis, J.; Asorey, J.; Avila, S.; et al. Dark Energy Survey Year 1 Results: Cosmological Constraints from Galaxy Clustering and Weak Lensing. arXiv 2017, arXiv:1708.01530.

33. Troxel, M.A.; MacCrann, N.; Zuntz, J.; Eifler, T.F.; Krause, E.; Dodelson, S.; Gruen, D.; Blazek, J.; Friedrich, O.; Samuroff, S.; et al. Dark Energy Survey Year 1 Results: Cosmological Constraints from Cosmic Shear. arXiv 2017, arXiv:1708.01538.

34. Dunsby, P.K.S.; Elizalde, E.; Goswami, R.; Odintsov, S.; Gomez, D.S. On the LCDM Universe in f(R) gravity. Phys. Rev. D 2010, 82, 023519. [CrossRef]

35. Deffayet, C.; Esposito-Farese, G.; Woodard, R.P. Nonlocal metric formulations of MOND with sufficient lensing. Phys. Rev. D 2011, 84, 124054. [CrossRef]

36. Newton, I. Four Letters from Sir Isaac Newton to Doctor Bentley; Dodsley, J., Ed.; Pall-Mall: London, UK, 1756.

37. Woodard, R.P. Ostrogradsky's theorem on Hamiltonian instability. Scholarpedia 2015, 10, 32243. [CrossRef]

38. Eliezer, D.A.; Woodard, R.P. The Problem of Nonlocality in String Theory. Nucl. Phys. B 1989, 325, 389-469. [CrossRef]

39. Brooker, D.J.; Tsamis, N.C.; Woodard, R.P. Analytic approximation for the primordial spectra of single scalar potential models and its use in their reconstruction. Phys. Rev. D 2017, 96, 103531. [CrossRef]

40. Brooker, D.J.; Tsamis, N.C.; Woodard, R.P. From Non-trivial Geometries to Power Spectra and Vice Versa. J. Cosmol. Astropart. Phys. 2018, 1804, 3. [CrossRef]

41. Salopek, D.S.; Bond, J.R.; Bardeen, J.M. Designing Density Fluctuation Spectra in Inflation. Phys. Rev. D 1989, 40, 1753-1788. [CrossRef]

42. Leonard, K.E.; Woodard, R.P. Graviton Corrections to Vacuum Polarization during Inflation. Class. Quant. Grav. 2014, 31, 015010. [CrossRef] 
43. Tsamis, N.C.; Woodard, R.P. The Structure of perturbative quantum gravity on a De Sitter background. Commun. Math. Phys. 1994, 162, 217-248. [CrossRef]

44. Woodard, R.P. De Sitter Breaking in Field Theory. In Deserfest: A Celebration of the Life and Works of Stanley Deser; Liu, J.T., Duff, M.J., Stelle, K.S., Woodard, R.P., Eds.; World Scientific: Hackensack, NJ, USA, 2006; pp. 339-351. [CrossRef]

45. Wang, C.L.; Woodard, R.P. Excitation of Photons by Inflationary Gravitons. Phys. Rev. D 2015, 91, 124054. [CrossRef]

46. Glavan, D.; Miao, S.P.; Prokopec, T.; Woodard, R.P. Electrodynamic Effects of Inflationary Gravitons. Class. Quant. Grav. 2014, 31, 175002. [CrossRef]

47. Tsamis, N.C.; Woodard, R.P. One loop graviton selfenergy in a locally de Sitter background. Phys. Rev. D 1996, 54, 2621-2639. [CrossRef]

48. Mora, P.J.; Tsamis, N.C.; Woodard, R.P. Hartree approximation to the one loop quantum gravitationalcorrection to the graviton mode function on de Sitter. J. Cosmol. Astropart. Phys. 2013, 1310, 018. [CrossRef]

49. Park, S.; Woodard, R.P. Scalar Contribution to the Graviton Self-Energy during Inflation. Phys. Rev. D 2011, 83, 084049. [CrossRef]

50. Park, S.; Woodard, R.P. Inflationary Scalars Don't Affect Gravitons at One Loop. Phys. Rev. D 2011, 84, 124058. [CrossRef]

51. Park, S.; Prokopec, T.; Woodard, R.P. Quantum Scalar Corrections to the Gravitational Potentials on de Sitter Background. J. High Energy Phys. 2016, 1601, 074. [CrossRef]

52. Tsamis, N.C.; Woodard, R.P. Quantum gravity slows inflation. Nucl. Phys. B 1996, 474, 235-248. [CrossRef]

53. Tsamis, N.C.; Woodard, R.P. A Gravitational Mechanism for Cosmological Screening. Int. J. Mod. Phys. D 2011, 20, 2847-2851. [CrossRef]

54. Weinberg, S. The Cosmological Constant Problem. Rev. Mod. Phys. 1989, 61, 1-23. [CrossRef]

55. Carroll, S.M. The Cosmological constant. Living Rev. Rel. 2001, 4, 1. [CrossRef] [PubMed]

56. Miao, S.P.; Woodard, R.P. The Fermion self-energy during inflation. Class. Quant. Grav. 2006, 23, $1721-1761$. [CrossRef]

57. Miao, S.P.; Woodard, R.P. Gravitons Enhance Fermions during Inflation. Phys. Rev. D 2006, 74, 024021. [CrossRef]

58. Prokopec, T.; Tornkvist, O.; Woodard, R.P. One loop vacuum polarization in a locally de Sitter background. Ann. Phys. 2003, 303, 251-274. [CrossRef]

59. Prokopec, T.; Tsamis, N.C.; Woodard, R.P. Two Loop Scalar Bilinears for Inflationary SQED. Class. Quant. Grav. 2007, 24, 201-230. [CrossRef]

60. Prokopec, T.; Tsamis, N.C.; Woodard, R.P. Two loop stress-energy tensor for inflationary scalar electrodynamics. Phys. Rev. D 2008, 78, 043523. [CrossRef]

61. Radkowski, A.F. Some Aspects of the Source Description of Gravitation. Ann. Phys. 1970, 56, 319. [CrossRef]

62. Detweiler, S.L. Pulsar timing measurements and the search for gravitational waves. Astrophys. J. 1979, 234, 1100-1104. [CrossRef]

63. Miao, S.P.; Prokopec, T.; Woodard, R.P. Deducing Cosmological Observables from the S-matrix. Phys. Rev. D 2017, 96, 104029. [CrossRef]

64. Miao, S.P.; Park, S. Alternate Definitions of Loop Corrections to the Primordial Power Spectra. Phys. Rev. D 2014, 89, 064053. [CrossRef]

65. Fröb, M.B. Gauge-invariant quantum gravitational corrections to correlation functions. Class. Quant. Grav. 2018, 35, 055006. [CrossRef]

66. Fröb, M.B.; Lima, W.C.C. Propagators for gauge-invariant observables in cosmology. Class. Quant. Grav. 2018, 35, 095010. [CrossRef]

67. Fröb, M.B.; Hack, T.P.; Khavkine, I. Approaches to linear local gauge-invariant observables in inflationary cosmologies. Class. Quant. Grav. 2018, 35, 115002. [CrossRef]

68. Tsamis, N.C.; Woodard, R.P. Pure Gravitational Back-Reaction Observables. Phys. Rev. D 2013, 88, 044040. [CrossRef]

69. Miao, S.P.; Tsamis, N.C.; Woodard, R.P. Invariant measure of the one-loop quantum gravitational backreaction on inflation. Phys. Rev. D 2017, 95, 125008. [CrossRef]

70. Christodoulou, D. Nonlinear nature of gravitation and gravitational wave experiments. Phys. Rev. Lett. 1991, 67, 1486-1489. [CrossRef] [PubMed] 
71. Ehrenberg, W.; Siday, R.E. The Refractive Index in Electron Optics and the Principles of Dynamics. Proc. Phys. Soc. 1949, B62, 8-21. [CrossRef]

72. Aharonov, Y.; Bohm, D. Significance of electromagnetic potentials in the quantum theory. Phys. Rev. 1959, 115, 485-491. [CrossRef]

73. Chambers, R.G. Shift of an Electron Interference Pattern by Enclosed Magnetic Flux. Phys. Rev. Lett. 1960, 5, 3-5. [CrossRef]

74. Giddings, S.B.; Sloth, M.S. Cosmological observables, IR growth of fluctuations, and scale-dependent anisotropies. Phys. Rev. D 2011, 84, 063528. [CrossRef]

75. Basu, S.; Woodard, R.P. Testing an Ansatz for the Leading Secular Loop Corrections from Quantum Gravity during Inflation. Class. Quant. Grav. 2016, 33, 205007. [CrossRef]

76. Basu, S.; Tsamis, N.C.; Woodard, R.P. Causality Implies Inflationary Back-Reaction. J. High Energy Phys. 2017, 1707, 037. [CrossRef]

77. Abramo, L.R.W.; Woodard, R.P. One loop back reaction on chaotic inflation. Phys. Rev. D 1999, 60, 044010. [CrossRef]

78. Abramo, L.R.; Woodard, R.P. No one loop back reaction in chaotic inflation. Phys. Rev. D 2002, 65, 063515. [CrossRef]

79. Parker, L.; Toms, D.J. Renormalization Group and Nonlocal Terms in the Curved Space-time Effective Action: Weak Field Results. Phys. Rev. D 1985, 32, 1409-1420. [CrossRef]

80. Banks, T. Prolegomena to a Theory of Bifurcating Universes: A Nonlocal Solution to the Cosmological Constant Problem Or Little Lambda Goes Back to the Future. Nucl. Phys. B 1988, 309, 493-512. [CrossRef]

81. Wetterich, C. Effective nonlocal Euclidean gravity. Gen. Rel. Grav. 1998, 30, 159-172. [CrossRef]

82. Barvinsky, A.O. Nonlocal action for long distance modifications of gravity theory. Phys. Lett. B 2003, 572, 109-116. [CrossRef]

83. Espriu, D.; Multamaki, T.; Vagenas, E.C. Cosmological significance of one-loop effective gravity. Phys. Lett. B 2005, 628, 197-205. [CrossRef]

84. Hamber, H.W.; Williams, R.M. Nonlocal effective gravitational field equations and the running of Newton's G. Phys. Rev. D 2005, 72, 044026. [CrossRef]

85. Biswas, T.; Mazumdar, A.; Siegel, W. Bouncing universes in string-inspired gravity. J. Cosmol. Astropart. Phys. 2006, 3, 9. [CrossRef]

86. Nacir, D.L.; Mazzitelli, F.D. Running of Newton's constant and non integer powers of the d'Alembertian. Phys. Rev. D 2007, 75, 024003. [CrossRef]

87. Khoury, J. Fading gravity and self-inflation. Phys. Rev. D 2007, 76, 123513. [CrossRef]

88. Capozziello, S.; Elizalde, E.; Nojiri, S.; Odintsov, S.D. Accelerating cosmologies from non-local higher-derivative gravity. Phys. Lett. B 2009, 671, 193-198. [CrossRef]

89. Biswas, T.; Koivisto, T.; Mazumdar, A. Towards a resolution of the cosmological singularity in non-local higher derivative theories of gravity. J. Cosmol. Astropart. Phys. 2010, 1011, 8. [CrossRef]

90. Zhang, Y.L.; Sasaki, M. Screening of cosmological constant in non-local cosmology. Int. J. Mod. Phys. D 2012, 21, 1250006. [CrossRef]

91. Barvinsky, A.O. Dark energy and dark matter from nonlocal ghost-free gravity theory. Phys. Lett. B 2012, 710 , 12-16. [CrossRef]

92. Barvinsky, A.O. Serendipitous discoveries in nonlocal gravity theory. Phys. Rev. D 2012, 85, 104018. [CrossRef]

93. Elizalde, E.; Pozdeeva, E.O.; Vernov, S.Y. De Sitter Universe in Non-local Gravity. Phys. Rev. D 2012, 85, 044002. [CrossRef]

94. Barvinsky, A.O.; Gusev, Y.V. New representation of the nonlocal ghost-free gravity theory. Phys. Part. Nucl. 2013, 44, 213-219. [CrossRef]

95. Biswas, T.; Conroy, A.; Koshelev, A.S.; Mazumdar, A. Generalized ghost-free quadratic curvature gravity. Class. Quant. Grav. 2014, 31, 015022; Erratum in 2014, 31, 159501. [CrossRef]

96. Foffa, S.; Maggiore, M.; Mitsou, E. Apparent ghosts and spurious degrees of freedom in non-local theories. Phys. Lett. B 2014, 733, 76-83. [CrossRef]

97. Foffa, S.; Maggiore, M.; Mitsou, E. Cosmological dynamics and dark energy from nonlocal infrared modifications of gravity. Int. J. Mod. Phys. A 2014, 29, 1450116. [CrossRef]

98. Rahvar, S.; Mashhoon, B. Observational Tests of Nonlocal Gravity: Galaxy Rotation Curves and Clusters of Galaxies. Phys. Rev. D 2014, 89, 104011. [CrossRef] 
99. Kehagias, A.; Maggiore, M. Spherically symmetric static solutions in a nonlocal infrared modification of General Relativity. J. High Energy Phys. 2014, 1408, 029. [CrossRef]

100. Maggiore, M.; Mancarella, M. Nonlocal gravity and dark energy. Phys. Rev. D 2014, 90, 023005. [CrossRef]

101. Dirian, Y.; Foffa, S.; Khosravi, N.; Kunz, M.; Maggiore, M. Cosmological perturbations and structure formation in nonlocal infrared modifications of general relativity. J. Cosmol. Astropart. Phys. 2014, 1406, 033. [CrossRef]

102. Conroy, A.; Koivisto, T.; Mazumdar, A.; Teimouri, A. Generalized quadratic curvature, non-local infrared modifications of gravity and Newtonian potentials. Class. Quant. Grav. 2015, 32, 015024. [CrossRef]

103. Barreira, A.; Li, B.; Hellwing, W.A.; Baugh, C.M.; Pascoli, S. Nonlinear structure formation in Nonlocal Gravity. J. Cosmol. Astropart. Phys. 2014, 1409, 031. [CrossRef]

104. Dirian, Y.; Mitsou, E. Stability analysis and future singularity of the $m^{2} R \square^{-2} R$ model of non-local gravity. J. Cosmol. Astropart. Phys. 2014, 1410, 065. [CrossRef]

105. Dirian, Y.; Foffa, S.; Kunz, M.; Maggiore, M.; Pettorino, V. Non-local gravity and comparison with observational datasets. J. Cosmol. Astropart. Phys. 2015, 1504, 044. [CrossRef]

106. Netto, T.D.P.; Pelinson, A.M.; Shapiro, I.L.; Starobinsky, A.A. From stable to unstable anomaly-induced inflation. Eur. Phys. J. C 2016, 76, 544. [CrossRef]

107. Zhang, X.; Wu, Y.B.; Li, S.; Liu, Y.C.; Chen, B.H.; Chai, Y.T.; Shu, S. Cosmological evolution of generalized non-local gravity. J. Cosmol. Astropart. Phys. 2016, 1607, 3. [CrossRef]

108. Cusin, G.; Foffa, S.; Maggiore, M.; Mancarella, M. Nonlocal gravity with a Weyl-square term. Phys. Rev. D 2016, 93, 043006. [CrossRef]

109. Zhang, Y.L.; Koyama, K.; Sasaki, M.; Zhao, G.B. Acausality in Nonlocal Gravity Theory. J. High Energy Phys. 2016, 1603, 039. [CrossRef]

110. Cusin, G.; Foffa, S.; Maggiore, M.; Mancarella, M. Conformal symmetry and nonlinear extensions of nonlocal gravity. Phys. Rev. D 2016, 93, 083008. [CrossRef]

111. Dirian, Y.; Foffa, S.; Kunz, M.; Maggiore, M.; Pettorino, V. Non-local gravity and comparison with observational datasets. II. Updated results and Bayesian model comparison with $\Lambda$ CDM. J. Cosmol. Astropart. Phys. 2016, 1605, 068. [CrossRef]

112. Maggiore, M. Perturbative loop corrections and nonlocal gravity. Phys. Rev. D 2016, 93, 063008. [CrossRef]

113. Koshelev, A.S.; Modesto, L.; Rachwal, L.; Starobinsky, A.A. Occurrence of exact $R^{2}$ inflation in non-local UV-complete gravity. J. High Energy Phys. 2016, 1611, 067. [CrossRef]

114. Nersisyan, H.; Akrami, Y.; Amendola, L.; Koivisto, T.S.; Rubio, J. Dynamical analysis of $R \frac{1}{\square^{2}} R \operatorname{cosmology:}$ Impact of initial conditions and constraints from supernovae. Phys. Rev. D 2016, 94, 043531. [CrossRef]

115. Maggiore, M. Nonlocal Infrared Modifications of Gravity. A Review. Fundam. Theor. Phys. 2017, 187, $221-281$. [CrossRef]

116. Wu, Y.B.; Zhang, X.; Wu, M.M.; Zhang, N.; Chen, B.H. Energy conditions and constraints on the generalized non-local gravity model. Chin. Phys. Lett. 2017, 34, 079801. [CrossRef]

117. Mashhoon, B. Nonlocal Gravity; Oxford University Press: Oxford, UK, 2017.

118. Romania, M.G.; Tsamis, N.C.; Woodard, R.P. Quantum Gravity and Inflation. Lect. Notes Phys. 2013, 863, 375-395. [CrossRef]

119. Tsamis, N.C.; Woodard, R.P. A Phenomenological Model for the Early Universe. Phys. Rev. D 2009, 80, 083512. [CrossRef]

120. Soussa, M.E.; Woodard, R.P. A Nonlocal metric formulation of MOND. Class. Quant. Grav. 2003, 20, $2737-2751$. [CrossRef]

121. Deser, S.; Woodard, R.P. Nonlocal Cosmology. Phys. Rev. Lett. 2007, 99, 111301. [CrossRef] [PubMed]

122. Deser, S.; Woodard, R.P. Observational Viability and Stability of Nonlocal Cosmology. J. Cosmol. Astropart. Phys. 2013, 1311, 036. [CrossRef]

123. Woodard, R.P. Nonlocal Models of Cosmic Acceleration. Found. Phys. 2014, 44, 213-233. [CrossRef]

124. Soussa, M.E.; Woodard, R.P. A Generic problem with purely metric formulations of MOND. Phys. Lett. B 2004, 578, 253-258. [CrossRef]

125. Tsamis, N.C.; Woodard, R.P. Primordial Density Perturbations and Reheating from Gravity. Phys. Rev. D 2010, 82, 063502. [CrossRef] 
126. Tsamis, N.C.; Woodard, R.P. Post-Inflationary Evolution via Gravitation. Phys. Rev. D 2010, 81, 103509. [CrossRef]

127. Tsamis, N.C.; Woodard, R.P. A Caveat on Building Nonlocal Models of Cosmology. J. Cosmol. Astropart. Phys. 2014, 1409, 8. [CrossRef]

128. Tsamis, N.C.; Woodard, R.P. Improved cosmological model. Phys. Rev. D 2016, 94, 043508. [CrossRef]

129. Deffayet, C.; Woodard, R.P. Reconstructing the Distortion Function for Nonlocal Cosmology. J. Cosmol. Astropart. Phys. 2009, 8, 023. [CrossRef]

130. Park, S.; Dodelson, S. Structure formation in a nonlocally modified gravity model. Phys. Rev. D 2013, 87, 024003. [CrossRef]

131. Dodelson, S.; Park, S. Nonlocal Gravity and Structure in the Universe. Phys. Rev. D 2014, 90, 043535. [CrossRef]

132. Park, S.; Shafieloo, A. Growth of perturbations in nonlocal gravity with non- $\Lambda$ CDM background. Phys. Rev. D 2017, 95, 064061. [CrossRef]

133. Nersisyan, H.; Cid, A.F.; Amendola, L. Structure formation in the Deser-Woodard nonlocal gravity model: A reappraisal. J. Cosmol. Astropart. Phys. 2017, 1704, 046. [CrossRef]

134. Park, S. Revival of the Deser-Woodard nonlocal gravity model: Comparison of the original nonlocal form and a localized formulation. Phys. Rev. D 2018, 97, 044006. [CrossRef]

135. Deffayet, C.; Esposito-Farese, G.; Woodard, R.P. Field equations and cosmology for a class of nonlocal metric models of MOND. Phys. Rev. D 2014, 90, 064038; Addendum: 2014, 90, 089901. [CrossRef]

136. Woodard, R.P. Nonlocal metric realizations of MOND. Can. J. Phys. 2015, 93, 242-249. [CrossRef]

137. Kim, M.; Rahat, M.H.; Sayeb, M.; Tan, L.; Woodard, R.P.; Xu, B. Determining Cosmology for a Nonlocal Realization of MOND. Phys. Rev. D 2016, 94, 104009. [CrossRef]

138. Tan, L.; Woodard, R.P. Structure Formation in Nonlocal MOND. J. Cosmol. Astropart. Phys. 2018, $1805,037$. [CrossRef]

(C) 2018 by the authors. Licensee MDPI, Basel, Switzerland. This article is an open access article distributed under the terms and conditions of the Creative Commons Attribution (CC BY) license (http:/ / creativecommons.org/licenses/by/4.0/). 